\title{
Simple Method for the Preparation of Composites Based on PA6 and Partially Exfoliated Graphite
}

\author{
Orietta Monticelli, ${ }^{1}$ Sergio Bocchini, ${ }^{2}$ Alberto Frache, ${ }^{2}$ Erika Simona Cozza, ${ }^{1}$ \\ Ornella Cavalleri, ${ }^{3}$ and Luigi Prati ${ }^{1}$ \\ ${ }^{1}$ Dipartimento di Chimica e Chimica Industriale, Università di Genova, Via Dodecaneso, 31, 16146 Genova, Italy \\ ${ }^{2}$ Dipartimento di Scienza dei Materiali e Ingegneria Chimica, Politecnico di Torino, Sede di Alessandria, \\ Viale Teresa Michel, 5, 15121 Alessandria, Italy \\ ${ }^{3}$ Dipartimento di Fisica, Università di Genova, Via Dodecaneso, 33, 16146 Genova, Italy
}

Correspondence should be addressed to Orietta Monticelli, orietta.monticelli@unige.it

Received 19 December 2011; Accepted 19 January 2012

Academic Editor: Sevan P. Davtyan

Copyright ( $\odot 2012$ Orietta Monticelli et al. This is an open access article distributed under the Creative Commons Attribution License, which permits unrestricted use, distribution, and reproduction in any medium, provided the original work is properly cited.

In the present work, the preparation of composite systems based on polyamide 6 (PA6) and exfoliated graphite was attempted by applying a simple procedure, which consists of a preliminary dispersion/exfoliation of graphite in the monomer, namely, $\varepsilon$ caprolactam (CL), and a subsequent polymerization of the above system. Atomic force microscopy (AFM) demonstrated specific interactions between CL and graphite surface. The dispersion of graphite in the monomer and polymer was assessed by scanning (SEM) and transmission (TEM) electron microscopy, while mechanical tests allowed to evaluate the influence of graphite on the polymer properties.

\section{Introduction}

Among the various nanofillers used in the field of nanostructured materials, graphene represents one of the most promising as demonstrated by the number of recent publications [1]. As far the preparation of graphene-based nanocomposite is concerned, the challenge is clearly the attainment of a fine dispersion of the above nanofiller in the polymer matrix. Indeed, while it is difficult to obtain homogeneous dispersions of graphene in polymer matrices, it is much easier to do it with single sheets of graphite oxide (GO), which contains hydroxyl and epoxy groups on the basal planes and carboxy groups on the hedges. As GO, due to its hydrophilic nature, can be easily exfoliated in aqueous media, nanocomposites have been created with GO and watersoluble polymers such as poly(ethylene oxide) (PEO) [2] or poly(vinyl alcohol) (PVA) [3]. Still, the main obstacle linked to GO incompatibility with most polymer systems remains. Thus, it is necessary to proceed to surface property modification of GO by functionalization in order to make easier its dispersion in polymer matrices. Indeed, using GO after chemical modification with isocyanate or amine, composites have also been produced in aprotic solvents with hydrophobic polymers such as polystyrene (PS) [4], polyurethane (PU) [5], or poly(methyl methacrylate) (PMMA) [6]. Moreover, the material electrical conductivity can be restored via chemical reduction of the graphene oxide. Another route generally used to disperse graphene into polymers is melt blending, being this latter economic and environmental friendly. Together with the above-mentioned approaches, as for other nanofillers, also in the case of graphene-based nanocomposite, the in situ polymerization has been applied. Indeed, successful polymerizations of poly(vinyl alcohol) (PVA) [7], poly(methyl methacrylate) (PMMA) [8], epoxy [9], poly (arylene disulfide) [10], polyethylene (PE) [11], and polypropylene (PP) [12] with graphene oxide or silicone foams [13] and PU [14] with thermally reduced graphene oxide have been reported.

As far the preparation of composites based on polyamide 6 (PA6) is concerned, object of the present work, only very few works have been reported so far. Indeed, recently Steurer et al. [15] reported on the preparation of nanocomposites 
based on thermally reduced graphite oxide and PA6 by melt blending, which showed improved stiffness and lower percolation threshold with respect to the nanocomposites containing conventional carbon nanoparticles. Melt blending approach was also applied by Fukushima et al. [16] to prepare PA6 composites containing exfoliated graphite flakes. Indeed, the addition of small amounts of the above filler showed a marked improvement in thermal and electrical conductivity of the composites. Also in the case of PA6, in situ polymerization approach has been applied by using graphene oxide. Indeed, recently, $\mathrm{Xu}$ and Gao [17] demonstrated that by a condensation reaction between the carboxylic acid groups on GO and terminal amino ends of PA6 chains, the macromolecular chains of PA6 were effectively grafted onto GO sheets.

However, in general the use of graphene oxide involves a previous oxidation of graphite and subsequent reduction of GO, in order to restore the material electrical conductivity. In this light, the development of methods able to disperse graphene in one step into a polymer matrix is a significant current research issue.

In the present work, the preparation of composite systems based on PA6 and exfoliated graphite has been attempted by applying a simple procedure, which consists of a preliminary dispersion/exfoliation of graphite in the monomer, $\varepsilon$-caprolactam, and a subsequent polymerization of the above system.

\section{Experimental}

2.1. Preparation of Composite Systems. $\varepsilon$-Caprolactam (CL) and graphite powder used were purchased from SigmaAldrich. The graphite was dispersed in CL (cylindrical vial, $20 \mathrm{~mL}$ of monomer) at different concentrations (from 0.1 to $1.0 \mathrm{wt} . \mathrm{-} \%$ ) by sonication in a sonic bath (Model Ney Ultrasonic) at $80^{\circ} \mathrm{C}$ for 240 minutes. The resultant dispersion was then centrifuged using a Hettich Mikro 22R centrifuge for 2 minutes at $600 \mathrm{rpm}$. In order to avoid the solidification of the monomer, the vial was heated up to $80^{\circ} \mathrm{C}$ and the centrifugation was repeated at least for four times. The precipitate was separate from the molten CL and the sonication was repeated for $60 \mathrm{~min}$. The dispersion was then centrifuged by applying the same procedure previously reported. In order to find the concentration after centrifugation, the precipitate was collected with acetone and passed through a polyvinylidene fluoride filters. Careful measurements of the filtered mass, gave the concentration of the dispersed phase after centrifugation.

Neat PA6 and composite systems were prepared by applying a classical hydrolytic polymerization.

2.2. Characterization. Tapping mode AFM measurements were performed using a Multimode/Nanoscope IV system (Digital Instruments-Bruker) and Si cantilevers (OMCLAC160TS, Olympus). During AFM measurements relative humidity was kept below 30\%. AFM measurements were performed on highly ordered pyrolytic graphite (HOPG) modified by CL. Indeed, CL was dissolved in Milli-Q water
(Millipore, resistivity $18 \mathrm{M} \Omega \mathrm{cm}$ ) to a final of $1 \mathrm{mM}$. Samples were prepared by depositing a drop of solution $(200 \mu \mathrm{L})$ onto fresly cleaved HOPG. After typically 1 hour adsorption, samples were thoroughly rinsed with MilliQ water and dried under a nitrogen flow. Samples were prepared and characterized at room temperature.

Solution viscosity of both neat PA6 and composite samples was measured in a suspended level Ubbelohde viscometer at $25^{\circ} \mathrm{C}$ in $96 \% \mathrm{H}_{2} \mathrm{SO}_{4}$.

A Leica Stereoscan 440 scanning electron microscope was used to examine the $\mathrm{CL} /$ graphite systems morphologies. All samples were thinly sputter-coated with gold using a Polaron E5100 sputter coater.

Transmission electron microscopy analyses were performed with a high-resolution equipment (JEOL 2010). The measurements were carried out using an accelerating voltage of $200 \mathrm{kV}$. Ultrathin sections of about $100 \mathrm{~nm}$ thick were cut with a Power TOMEX microtome equipped with a diamond knife and placed on a 200-mesh copper grid. Differential scanning calorimetry was performed under a continuous nitrogen purge on a Mettler calorimetric apparatus, mod. TC10A. Both calibrations of heat flow and temperature were based on a run in which one standard sample (indium) was heated through its melting point. Samples having a mass between 2.5 and $11 \mathrm{mg}$ were used. Data were gathered using a scan rate of $10^{\circ} \mathrm{C} / \mathrm{min}$.

Mechanical tests were performed with DMA Q800 TA Instruments with an elongation rate of $1 \% / \mathrm{min}$ at $25^{\circ} \mathrm{C}$ with a preload of $0.01 \mathrm{~N}$. The samples were prepared by cutting strips from the films with a width of $6 \mathrm{~mm}$, a height of $26 \mathrm{~mm}$, and a thickness of about $0.4 \mathrm{~mm}$ prepared by hot compression molding with $5 \mathrm{MPa}$ at $230^{\circ} \mathrm{C}$ for $1 \mathrm{~min}$. For each material at list three samples were characterized.

\section{Results and Discussion}

The work on the preparation of composites based on PA6 has been preliminary focused on the study of graphite dispersion/exfoliation in the monomer, that is, $\varepsilon$-caprolactam $(\mathrm{CL})$. Indeed, recently, by considering the analogy between carbon nanotubes and graphene, Hernandez et al. [19] demonstrated a scalable, high yield method to produce high-quality, unoxidized graphene from powdered graphite. By using organic solvents such as $\mathrm{N}$-methylpyrrolidone, $\mathrm{N}, \mathrm{N}$-dimethylacetamide, and $\gamma$-butyrolactone, graphene can be dispersed. This occurs because the energy required to exfoliate graphene is balanced by the solvent-graphene interactions for solvents whose surface energy matches that of graphene. $\mathrm{CL}$ was therefore chosen as solvent since its surface tension $\left(30.4 \mathrm{~mJ} / \mathrm{m}^{2}\right)$ is in the range of that of the best solvents, capable to keep the highest concentration of graphite/graphene after centrifugation.

Prior to graphite exfoliation experiments, we performed preliminary measurements aimed to investigate the interactions between CL and the graphite surface. AFM was employed to evaluate the occurrence of structural changes in the highly ordered pyrolytic graphite (HOPG) surface structure 


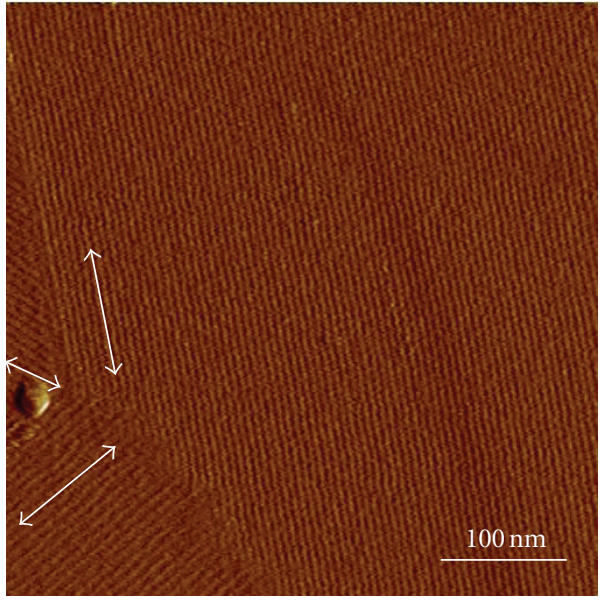

(a)

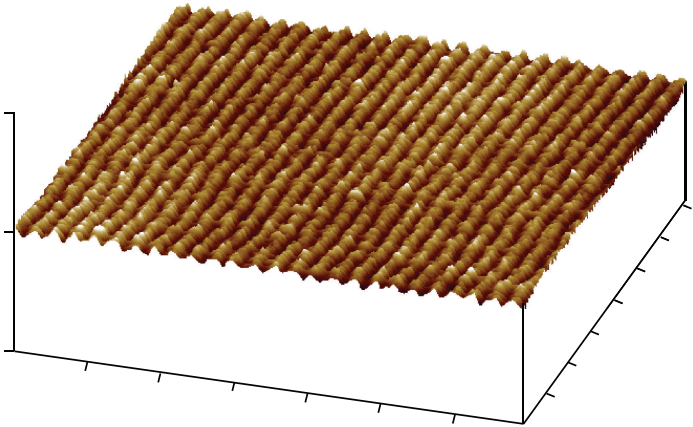

(b)

FIgURE 1: (a) Amplitude tapping mode AFM image of a HOPG sample after interaction with the CL solution. White arrows indicate the orientations of adjacent patterned domains. (b) 3D rendering of a height tapping mode AFM image of the $6.2 \mathrm{~nm}$ patterned domain. Image size: $150 \mathrm{~nm} \times 150 \mathrm{~nm}, z$-scale: $2.5 \mathrm{~nm}$.

TABLE 1: Characteristics of the sample prepared.

\begin{tabular}{|c|c|c|c|c|c|c|c|}
\hline Sample code & $\begin{array}{c}\text { Initial graphite } \\
\text { concentration in CL } \\
\text { (wt.-\%) }\end{array}$ & $\begin{array}{l}\text { Remaining graphite } \\
\text { concentration in CL } \\
\text { (wt.-\%) }\end{array}$ & $M_{w} \cdot 10^{-3^{\mathrm{a}}}$ & $T_{g}\left({ }^{\circ} \mathrm{C}\right)$ & $x_{c}^{b}(\%)$ & $T_{c}\left({ }^{\circ} \mathrm{C}\right)$ & $T_{m}\left({ }^{\circ} \mathrm{C}\right)$ \\
\hline PA6 & - & - & 30 & 50 & 45 & 196 & 218 \\
\hline PA6(1.0) & 1.0 & 0.1 & 29 & 50 & 44 & 195 & 218 \\
\hline PA6(0.5) & 0.5 & 0.06 & 28 & 49 & 45 & 196 & 219 \\
\hline PA6(0.2) & 0.2 & 0.02 & 30 & 51 & 46 & 196 & 218 \\
\hline
\end{tabular}

Glass transition temperature $\left(T_{g}\right)$, melting temperature $\left(T_{m}\right)$, crystallization temperature $\left(T_{c}\right)$, and degree of crystallinity $\left(x_{c}\right)$ were determined from the second scan.

${ }^{\mathrm{a}} M_{w}$ was calculated by the following equation: $[\eta]=0,51 \cdot 10^{-3} M_{w}^{0.74}[18]$ by measuring $[\eta]$ in $\mathrm{H}_{2} \mathrm{SO}_{4}$ at $20^{\circ} \mathrm{C}$.

upon interaction with CL. Since CL is solid at room temperature, an aqueous solution of CL was used.

As shown in the AFM image in Figure 1, the interactions of the CL solution with the HOPG surface leads to the formation of extended extremely regular nanopatterned domains, formed by parallel stripes. These domains, characterized by a $6.2 \pm 0.2 \mathrm{~nm}$ periodicity, are oriented according to the threefold symmetry of HOPG. In regions where discontinuities between adjacent domains can be observed, it has been possible to measure the thickness of the patterned layer which is found to be of $0.35 \pm 0.05 \mathrm{~nm}$, in good agreement with the graphite interplane distance. Based on several experimental findings [20], the nanopattern formation can be regarded as a consequence of the interaction between the CL solution and the graphite surface: the interactions between the solution and the HOPG basal plane could result into a weakening of the interactions between the topmost graphite layers, leading to a weakly bound "graphene-like" layer which subsequently undergoes a rippling process in a similar way as reported for both supported and free-standing graphene [21-23]. Once verified that the CL solution can interact with the graphite surface and can influence the HOPG interplane interactions, graphite exfoliation has been attempted in the above monomer by applying an ultrasonic treatment. Indeed, the sonication, carried out at $80^{\circ} \mathrm{C}$, namely, above the melting temperature of the monomer, allows to obtain a grey liquid consisting of a homogenous phase and macroscopic aggregates, which can be removed by centrifugation.

The concentration of the dispersed phase after the centrifugation for samples containing different amounts of graphite $(0.1,0.5$ and 1.0 wt.-\%), which has been calculated by measuring the filtered mass, has been reported in Table 1.

It comes out that graphite can be dispersed at a concentration up to $0.1 \mathrm{wt} .-\%$. The state of the material remaining dispersed in the monomer has been investigated by SEM analysis.

Figure 2 shows a SEM micrograph of the system CL/graphene prepared by using an initial graphite concentration of 0.5 wt. $\%$. It is clearly evident on the surface of the sample almost transparent graphite aggregates, demonstrating a fine dispersion in the monomer.

It is relevant to point out that graphite dispersion is strongly related with the sonication time $\left(t_{s}\right)$, parameter which is generally not considered during the process. Indeed, while the concentration of the dispersed phase increases with increasing $t_{s}$, graphite layer dimension decrease. The dimension of the aggregates passes from $0.5 \div 2 \mu \mathrm{m}$ applying a sonication time of 4 hours to $0.1 \div 0.3 \mu \mathrm{m}$ by increasing 
TABLE 2: Results of mechanical tests.

\begin{tabular}{lcccc}
\hline & Modulus (Mpa) & Strain at break (\%) & Max yield stress (Mpa) & Stress at break (Mpa) \\
\hline PA6 & 3275 & 0.7 & - & 17 \\
PA6(0.5) & 1820 & 1.2 & - & 20 \\
PA6(1.0) & 2087 & 26.0 & 16 & 11 \\
\hline
\end{tabular}

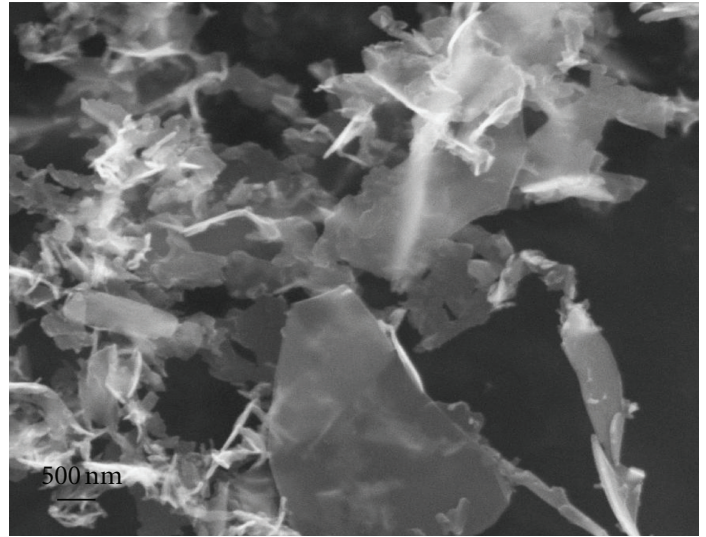

FIGURE 2: SEM micrograph of the graphite (initial graphite concentration $0.5 \mathrm{wt} .-\%) /$ monomer system after sonication and centrifugation.

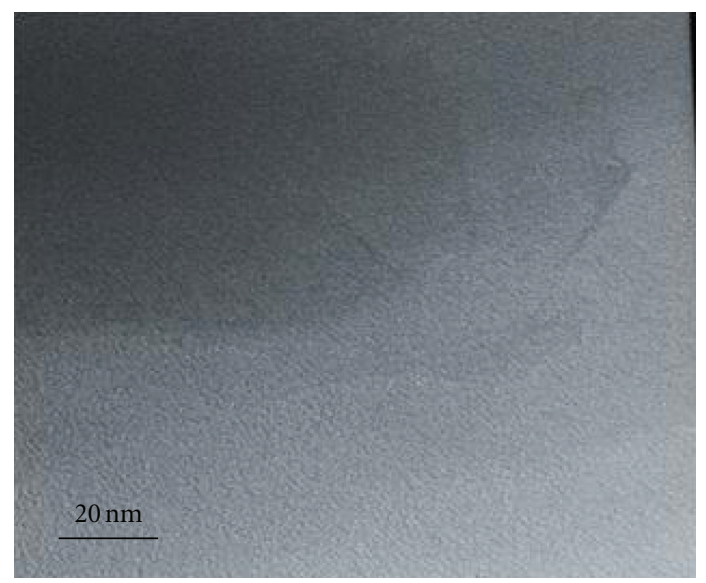

Figure 3: TEM micrograph of the sample PA6(0.5).

$t_{s}$ up to $8 \mathrm{~h}$. In this light, in order to preserve graphite layer size, the nanofiller dimension influencing the final material properties, a sonication time of 4 hours has been chosen. The so prepared CL/graphite systems underwent a polymerization process.

In order to get insight into the graphite dispersion in the polymer, TEM analyses were carried out.

TEM micrograph of the sample PA6(0.5), reported in Figure 3, demonstrates that together with aggregates also few-layer graphite is present in the polymer matrix. In particular, by analyzing the flake edges, these objects turn out to be formed by only two-three layers. It is worth underlining that similar results have been found also for the samples containing a lower and a higher concentration of graphite, namely, PA6(0.2) and PA6(1.0).

As reported in Table 1, the presence of graphite does not influence the molecular mass and thermal properties of the polymer matrix, but it has been found to modify its mechanical properties.

Because of the low quantity available, the mechanical properties were performed on thin films using the DMTA instrument in a stress strain configuration and isothermal conditions. Although these results cannot be compared with a standard mechanical test, they could be useful to understand the specific action of graphene on PA6 properties. As shown in Table 2, while pristine PA6 exhibits a modulus at $25^{\circ} \mathrm{C}$ of $3300 \mathrm{MPa}, \mathrm{PA} 6(0.5)$ and PA6(1.0) show a modulus of $1800 \mathrm{MPa}(-45 \%)$ and $2100 \mathrm{MPa}(-36 \%)$, respectively. Moreover, the strain at break of PA6(0.5) increases from $0.7 \%$ (neat PA6) to $1.2 \%$. A more relevant increase in strain at break has been observed in the case of the sample PA6(1.0), being its strain at break of $26 \%$. In this light, it is possible to conclude that the sample with the highest concentration of exfoliated graphite, behaves as a more plastic material. Taking into account that the molecular masses of the different samples are similar and their $T_{g}$ do not change, no plasticizing related to $M_{w}$ can be supposed. Thus, it is possible to hypothesize that the peculiar mechanical behavior of the sample PA6(1.0) is due to a plasticizing effect, which is related to weak interactions between the nanofiller and the polymer [24]. Moreover, these results seem to support the graphene lubrificant effect found recently in nanocomposites based on hydrogels [25].

\section{Conclusions}

In conclusion, composites based on PA6 and partially exfoliated graphite have been prepared by in situ polymerization, starting from the direct graphite exfoliation in $\varepsilon$-caprolactam (CL). Indeed, specific interactions between graphite surface and the above monomer have been assessed by AFM measurements. By tuning the dispersion conditions of graphite in $\mathrm{CL}$, it is possible to partially exfoliate graphite, without breaking the layers. Moreover, the nanofiller shows a lubrificant effect, increasing in a relevant way the strain at break of the polymer matrix.

\section{Acknowledgment}

The present study was financed by MIUR under the scheme of PRIN 2008 projects. 


\section{References}

[1] M. J. Allen, V. C. Tung, and R. B. Kaner, "Honeycomb carbon: a review of graphene," Chemical Reviews, vol. 110, no. 1, pp. 132-145, 2010.

[2] Y. Matsuo, K. Tahara, and Y. Sugie, "Structure and thermal properties of poly(ethylene oxide)-intercalated graphite oxide," Carbon, vol. 35, no. 1, pp. 113-120, 1997.

[3] M. Hirata, T. Gotou, S. Horiuchi, M. Fujiwara, and M. Ohba, "Thin-film particles of graphite oxide 1: high-yield synthesis and flexibility of the particles," Carbon, vol. 42, no. 14, pp. 2929-2937, 2004.

[4] S. Stankovich, D. A. Dikin, G. H. B. Dommett et al., "Graphene-based composite materials," Nature, vol. 442, no. 7100, pp. 282-286, 2006.

[5] H. Kim, Y. Miura, and C. W. MacOsko, "Graphene/polyurethane nanocomposites for improved gas barrier and electrical conductivity," Chemistry of Materials, vol. 22, no. 11, pp. 34413450, 2010.

[6] B. Das, K. Eswar Prasad, U. Ramamurty, and C. N. R. Rao, "Nano-indentation studies on polymer matrix composites reinforced by few-layer graphene," Nanotechnology, vol. 20, no. 12, Article ID 125705, 2009.

[7] P. Liu, K. Gong, P. Xiao, and M. Xiao, "Preparation and characterization of poly(vinyl acetate)-intercalated graphite oxide nanocomposite," Journal of Materials Chemistry, vol. 10, no. 4, pp. 933-935, 2000.

[8] J. Y. Jang, M. S. Kim, H. M. Jeong, and C. M. Shin, "Graphite oxide/poly(methyl methacrylate) nanocomposites prepared by an novel method utilizing macroazoinitiator," Composites Science and Technology, vol. 69, p. 186, 2009.

[9] S. Wang, M. Tambraparni, J. Qiu, J. Tipton, and D. Dean, "Thermal expansion of graphene composites," Macromolecules, vol. 42, no. 14, pp. 5251-5255, 2009.

[10] X. S. Du, M. Xiao, Y. Z. Meng, and A. S. Hay, "Direct synthesis of poly(arylenedisulfide)/carbon nanosheet composites via the oxidation with graphite oxide," Carbon, vol. 43, no. 1, pp. 195-197, 2005.

[11] Y. Huang, Y. Qin, Y. Zhou, H. Niu, Z.-Z. Yu, and J.-Y. Dong, "Polypropylene/graphene oxide nanocomposites prepared by in situ ziegler-natta polymerization," Chemistry of Materials, vol. 22, no. 13, pp. 4096-4102, 2010.

[12] F. D. C. Fim, J. M. Guterres, N. R. S. Basso, and G. B. Galland, "Polyethylene/graphite nanocomposites obtained by in situ polymerization," Journal of Polymer Science, Part A, vol. 48, no. 3, pp. 692-698, 2010.

[13] R. Verdejo, F. Barroso-Bujans, M. A. Rodriguez-Perez, J. A. De Saja, and M. A. Lopez-Manchado, "Functionalized graphene sheet filled silicone foam nanocomposites," Journal of Materials Chemistry, vol. 18, no. 19, pp. 2221-2226, 2008.

[14] Y. R. Lee, A. V. Raghu, H. M. Jeong, and B. K. Kim, "Properties of waterborne polyurethane/functionalized graphene sheet nanocomposites prepared by an in situ method," Macromolecular Chemistry and Physics, vol. 210, no. 15, pp. 1247-1254, 2009.

[15] P. Steurer, R. Wissert, R. Thomann, and R. Mülhaupt, "Functionalized graphenes and thermoplastic nanocomposites based upon expanded graphite oxide," Macromolecular Rapid Communications, vol. 30, no. 4-5, pp. 316-327, 2009.

[16] H. Fukushima, L. T. Drzal, B. P. Rook, and M. J. Rich, "Thermal conductivity of exfoliated graphite nanocomposites," Journal of Thermal Analysis and Calorimetry, vol. 85, no. 1, pp. 235-238, 2006.
[17] Z. Xu and C. Gao, "In situ polymerization approach to graphene-reinforced nylon-6 composites," Macromolecules, vol. 43, no. 16, pp. 6716-6723, 2010.

[18] K. Ueda, M. Nakai, K. Hattori, K. Yamada, and K. Tai, "Synthesis of high molecular weight nylon 6 by anionic polymerization of $\varepsilon$-caprolactam: evaluation of molecular weight and molecular weight distribution," Kobunshi Ronbunshu, vol. 54, no. 6, pp. 401-406, 1997.

[19] Y. Hernandez, V. Nicolosi, M. Lotya et al., "High-yield production of graphene by liquid-phase exfoliation of graphite," Nature Nanotechnology, vol. 3, no. 9, pp. 563-568, 2008.

[20] A. Penco, T. Svaldo-Lanero, M. Prato et al., Graphite Nanopatterning through Interaction with Bio-Organic Molecules, Carbon Nanostructures, GraphITA2011, Springer, 2011.

[21] J. C. Meyer, A. K. Geim, M. I. Katsnelson, K. S. Novoselov, T. J. Booth, and S. Roth, "The structure of suspended graphene sheets," Nature, vol. 446, no. 7131, pp. 60-63, 2007.

[22] A. L. Vázquez de Parga, F. Calleja, B. Borca et al., "Periodically rippled graphene: growth and spatially resolved electronic structure," Physical Review Letters, vol. 100, no. 5, Article ID 056807, 4 pages, 2008.

[23] A. Locatelli, K. R. Knox, D. Cvetko et al., "Corrugation in exfoliated graphene: an electron microscopy and diffraction study," ACS Nano, vol. 4, no. 8, pp. 4879-4889, 2010.

[24] A. Sanz, M. Ruppel, J. F. Douglas, and J. T. Cabral, "Plasticization effect of $\mathrm{C}_{60}$ on the fast dynamics of polystyrene and related polymers: an incoherent neutron scattering study," Journal of Physics Condensed Matter, vol. 20, no. 10, 2008.

[25] V. Alzari, D. Nuvoli, S. Scognamillo et al., "Graphene-containing thermoresponsive nanocomposite hydrogels of poly $(\mathrm{N}-$ isopropylacrylamide) prepared by frontal polymerization," Journal of Materials Chemistry, vol. 21, no. 24, pp. 8727-8733, 2011. 

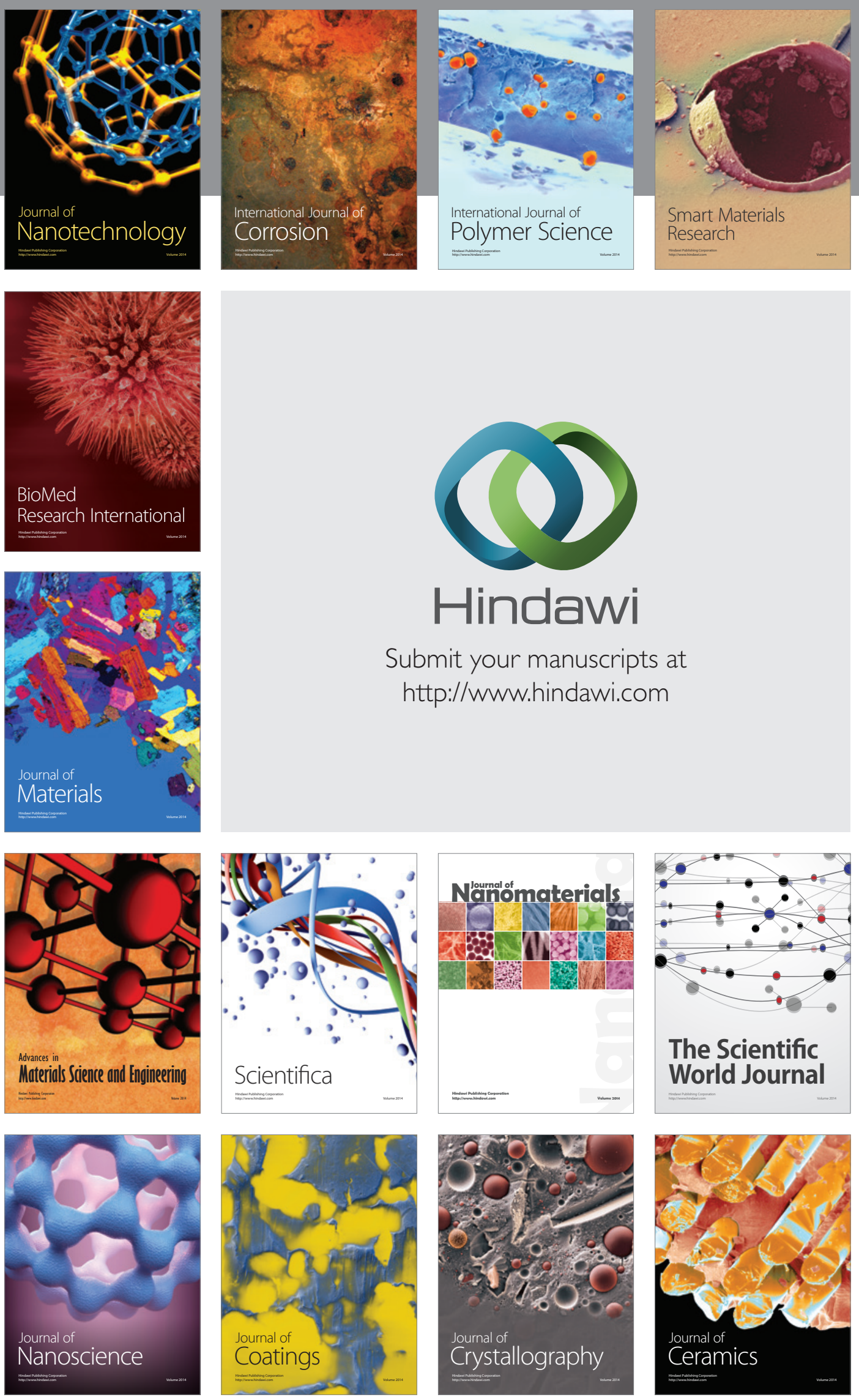

The Scientific World Journal

Submit your manuscripts at

http://www.hindawi.com

\section{World Journal}

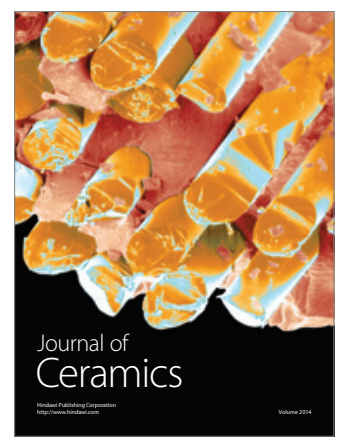

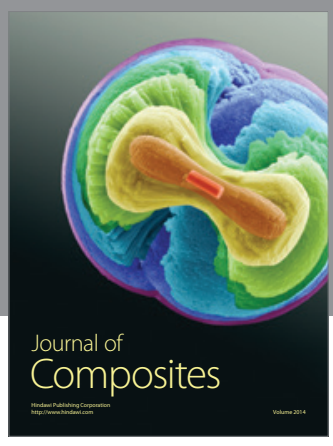
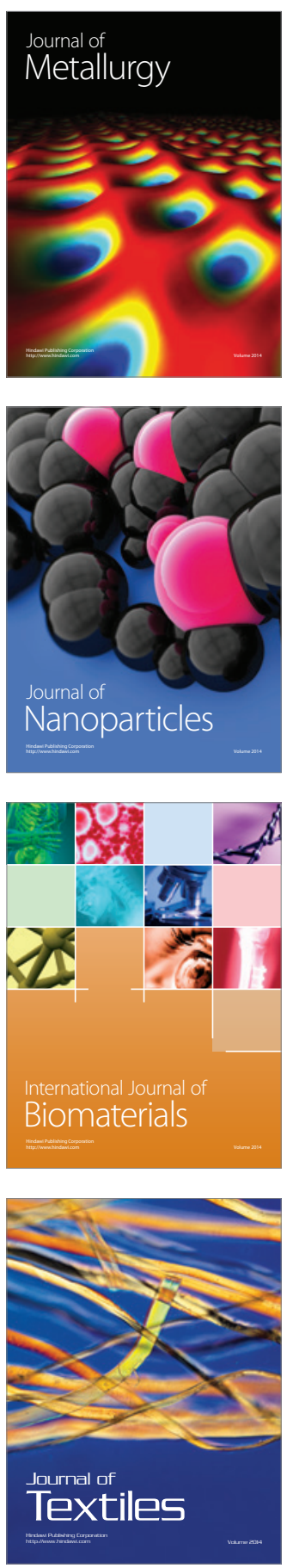